International Journal of Pure and Applied Mathematics

Volume 106 No. 4 2016, 1127-1150

ISSN: 1311-8080 (printed version); ISSN: 1314-3395 (on-line version)

url: http://www.ijpam.eu

doi: $10.12732 /$ ijpam.v106i4.14

\title{
SOLUTION OF HEAT CONDUCTION PROBLEM WITH DISCONTINUOUS BOUNDARY CONDITIONS IN NONHOMOGENOUS MOVING CYLINDER USING MAPLE
}

\author{
G.R. Gasimov ${ }^{1}$, Z.A. Abusutash ${ }^{2}$ \\ ${ }^{1}$ Faculty of Applied Mathematics \\ Baku State University \\ 23, Zahid Khalil, Baku, AZERBAIJAN \\ ${ }^{2}$ Department of Mathematics \\ Faculty of Science \\ Damietta University \\ New Damietta, 34517, EGYPT
}

\begin{abstract}
This paper presents a heat conduction problem with discontinuous boundary conditions in nonhomogeneous moving entire cylinder, which moves on the axis oz with any movement law $z=S(t)$. The temperature field is determined in the cylindrical coordinates system linked with motionless cylinder as a system in a single movement. Using the method inversion of the unit function $\eta\left(t-S^{-1}(z)\right)$, method a sequence of integral transformations like Fourier transform with respect to $z$, Hankel transform with respect to $r$, Bessel functions theory,general integral transforms theory, a solution in the form of the series is obtained.

And in order to illustrate theoretical results in this paper,we wrote special programming in Maple program and for a special cases, where numerical solutions were presented with explained graphics and discussed.
\end{abstract}

AMS Subject Classification: 35K05, 35A22, 33C10, 44A05, 42A38, 68N15

Key Words: heat conduction problem, Maple program, general integral transforms theory, sequential integral transformation method,bessel functions theory

Received: December 11, 2015

Published: March 13, 2016

$\S$ Correspondence author
(C) 2016 Academic Publications, Ltd.

url: www.acadpubl.eu 


\section{Introduction}

The thermal properties of a solid very with position, exact solutions can be found in a limited number of special,in this paper studying the case of one of these cases. Thermal conductivity problem of the semi-infinite nonhomogeneous solids, it has been studied by Carslaw and Jaeger [2]. Combining the traditional presentation of fundamental mathematical concepts with the contemporary computational benefits of Maple software submitted by Articolo [4]. In recent years, a number of the papers investigated analytical heat flow initiate within a hollow infinite cylinder using Maple program and the numerical solutions obtained and showed graphic animation, has been submitted by Shahout et al [7]. Solution of the problems thermal conductivity of the homogeneous moving bodies with finite size is obtained by Lotarev [8].Kholodovskii [9] considers boundary value problems for linear differential in piecewise homogenous cylinders into half cylinders by multilayer film. Studied thermal conductivity in the moving composite cylinder is obtained by [6]. Our motivation in this paper is to investigate the solution of heat conduction problem in physically nonhomogeneous moving entire cylinder, on the direction of the radius with discontinuous boundary conditions on the surface of the cylinder, which moves along the axis oz. Using the method insertion of the unit function,general integral transforms theory, method sequential of integral transformations like Fourier and Hankel transformation with corresponding inverse transformations respectively, a solution to the problem of the spread heat in the form of the series is obtained.As numerical solutions of the problem under study are achieved by using Maple18 package, where results are obtained and clear graphics with discussion. And these problems have a great importance in many engineering fields which intervention in the design of internal combustion engines, material in aviation, and the factories of the production of military.

\section{Formulation of the Problem}

Consider the temperature field of moving cylinder with discontinuous boundary conditions and initially at temperature $\varphi(r, z)$ for entire cylinder, which moves along the z-axis with any movement law.The temperature field is determined in the cylindrical coordinates system linked with motionless cylinder as a system in a single movement. This coordinate system moves from one environment in other environment with different thermophyscial descriptions. 
The formulation of this problem is given as:

$$
\begin{aligned}
& \frac{\partial}{\partial r}\left(k(r) \frac{\partial}{\partial r} T(r, z, t)\right)+\frac{\partial^{2}}{\partial z^{2}} T(r, z, t)-\rho c \frac{\partial}{\partial t} T(r, z, t)=0, \\
& 0<r<a, \quad 0<z<\ell, z=S(t), \quad t>0 \\
& T(r, z, 0)=\varphi(r, z), \\
& {\left.\left[\alpha_{1} \frac{\partial}{\partial r} T+\alpha_{2} T\right]\right|_{r=a}= \begin{cases}f_{0}(z, t), & \text { if } z<S(t), \\
f_{1}(z, t), & \text { if } z>S(t),\end{cases} } \\
& \left.T(r, z, t)\right|_{r=0}<\infty \\
& {\left.\left[\beta_{1} \frac{\partial}{\partial z} T-\beta_{2} T\right]\right|_{z=0}=-g_{0}(r, t),\left.\quad\left[\beta_{3} \frac{\partial}{\partial z} T+\beta_{4} T\right]\right|_{z=\ell}=g_{1}(r, t),} \\
& k(r)=k_{0} r^{m}\left(k_{0}-\text { const }, 0 \leq m \leq 1\right), \quad \rho \text { - const, } c-\text { const }, \\
& \alpha_{1}^{2}+\alpha_{2}^{2}>0, \beta_{1}^{2}+\beta_{2}^{2}>0, \beta_{3}^{2}+\beta_{4}^{2}>0, \beta_{2}^{2}+\beta_{4}^{2}>0,
\end{aligned}
$$

where $a$ is the radius of the cylinder.T, $k(r), \rho$ and $c$ are the temperature, thermal conductivity, density and specific heat of the cylinder, and $\alpha_{1}, \alpha_{2}, \beta_{1}, \beta_{2}$, $\beta_{3}, \beta_{4}$ are the coefficient of surface heat transfer are constants, and $\varphi(r, z), f_{0}(z, t)$, $f_{1}(z, t), g_{0}(r, t), g_{1}(r, t)$ are given functions.

\section{Solving Method}

The boundary condition (3) is discontinuous, we can write its as follows:

$$
\left.\left[\alpha_{1} \frac{\partial}{\partial r} T+\alpha_{2} T\right]\right|_{r=a}=f_{1}(z, t)-f_{2}(z, t) \eta\left(t-S^{-1}(z)\right) \equiv f(z, t) ;
$$

where

$$
\eta(\tau)= \begin{cases}1, & \text { if } \tau>0, \\ 0, & \text { if } \tau<0,\end{cases}
$$

$S^{-1}(z)$ is inverse function to $z=S(t), f_{2}(z, t)=f_{1}(z, t)-f_{0}(z, t)$. 
We choose a solution of this problem in the form:

$$
T(r, z, t)=T_{0}(r, z, t)+T_{1}(r, t)+T_{2}(r, t) z,
$$

where $T_{0}(r, z, t)$ satisfies homogeneous boundary conditions as follows:

$$
\left.\left[\beta_{1} \frac{\partial}{\partial z} T_{0}-\beta_{2} T_{0}\right]\right|_{z=0}=0,\left.\quad\left[\beta_{3} \frac{\partial}{\partial z} T_{0}+\beta_{4} T_{0}\right]\right|_{z=\ell}=0 .
$$

Using (7) and boundary conditions (5) we get:

$$
\begin{aligned}
& {\left.\left[\beta_{1}\left(\frac{\partial}{\partial z} T_{0}+T_{2}\right)-\beta_{2}\left(T_{0}+T_{1}+T_{2} z\right)\right]\right|_{z=0}=-g_{0}(r, t),} \\
& {\left[\beta_{3}\left(\frac{\partial}{\partial z} T_{0}+T_{2}\right)+\left.\beta_{4}\left(T_{0}+T_{1}+T_{2} z\right]\right|_{z=\ell}=g_{1}(r, t) .\right.}
\end{aligned}
$$

This system (9) we rewrite as following

$$
\begin{aligned}
& {\left.\left[\beta_{1} \frac{\partial}{\partial z} T_{0}-\beta_{2} T_{0}\right]\right|_{z=0}=\left(\beta_{2} T_{1}-\beta_{1} T_{2}-g_{0}\right),} \\
& {\left.\left[\beta_{3} \frac{\partial}{\partial z} T_{0}+\beta_{4} T_{0}\right]\right|_{z=\ell}=\left(-\beta_{4} T_{1}-\left(\beta_{4} \ell+\beta_{3}\right) T_{2}+g_{1}\right) .}
\end{aligned}
$$

In order to satisfies conditions of (8), the most $T_{1}, T_{2}$ satisfies the following system:

$$
\begin{aligned}
& \beta_{2} T_{1}-\beta_{1} T_{2}=g_{0}, \\
& \beta_{4} T_{1}+\left(\beta_{4} \ell+\beta_{3}\right) T_{2}=g_{1} .
\end{aligned}
$$

The determinant this system

$$
\Delta_{1}=\left|\begin{array}{ll}
\beta_{2} & -\beta_{1} \\
\beta_{4} & \beta_{4} \ell+\beta_{3}
\end{array}\right| ;
$$

also $\Delta_{1}=\beta_{1} \beta_{4}+\beta_{2}\left(\beta_{4} \ell+\beta_{3}\right) \neq 0$, we find that the system (11) with respect to $T_{1}, T_{2}$ there are non- trivial solution:

$$
\begin{aligned}
& T_{1}(r, t)=\frac{1}{\Delta_{1}}\left[g_{0}(r, t)\left(\beta_{4} \ell+\beta_{3}\right)+\beta_{1} g_{1}(r, t)\right], \\
& T_{2}(r, t)=\frac{1}{\Delta_{1}}\left[\beta_{2} g_{1}(r, t)-\beta_{4} g_{0}(r, t)\right] .
\end{aligned}
$$

Now we use integral transform with respect to $z$; on the basis of the theory of integral transformations [5], the kernel of this transformation assignment as solution of Sturm- Liouville problem in the following form:

$$
\frac{d^{2}}{d z^{2}} K(z)+\gamma^{2} K=0, \quad 0<z<\ell
$$




$$
\left.\left[\beta_{1} \frac{d}{d z} K-\beta_{2} K\right]\right|_{z=0}=0,\left.\quad\left[\beta_{3} \frac{d}{d z} K+\beta_{4} K\right]\right|_{z=\ell}=0 .
$$

The general solution of (12) is

$$
K(z)=C_{1} \cos \gamma z+C_{2} \sin \gamma z
$$

where $C_{1}$ and $C_{2}$ are arbitrary constants, from the condition (2), we can be found $C_{1}$ and $C_{2}$ as

follows:

$$
\begin{aligned}
& -\beta_{2} C_{1}+\beta_{1} \gamma C_{2}=0, \\
& \left(-\beta_{3} \gamma \sin \gamma \ell+\beta_{4} \cos \gamma \ell\right) C_{1}+\left(\beta_{3} \gamma \cos \gamma \ell+\beta_{4} \sin \gamma \ell\right) C_{2}=0 .
\end{aligned}
$$

For the existence non trivial solution to this system a necessary and sufficient condition the determinant of this system equal to zero:

$$
\left|\begin{array}{ll}
-\beta_{2} & \beta_{1} \gamma \\
-\beta_{3} \gamma \sin \gamma \ell+\beta_{4} \cos \gamma \ell & \beta_{3} \gamma \cos \gamma \ell+\beta_{4} \sin \gamma \ell
\end{array}\right|=0
$$

For parameter $\gamma$ we get the equation as follows:

$$
\left(\beta_{1} \beta_{4}+\beta_{2} \beta_{3}\right) \gamma \cos \gamma \ell=\left(\beta_{1} \beta_{3} \gamma^{2}-\beta_{2} \beta_{4}\right) \sin \gamma \ell
$$

or

$$
\operatorname{tg} \gamma \ell=\frac{\gamma\left(\beta_{1} \beta_{4}+\beta_{2} \beta_{3}\right)}{\gamma^{2} \beta_{1} \beta_{3}-\beta_{2} \beta_{4}} .
$$

The roots of (15) are all real, simple, and have an arithmetic numbers: $\gamma_{n}, n=$ $1,2, \ldots$ are the eigenvalues of the problem (12)-(13), and the corresponding solutions are eigenfunctions for this problem.

Assign the eigenfunctions of the first equation for the system (14)

$$
-\beta_{2} C_{1}+\beta_{1} \gamma_{n} C_{2}=0
$$

from here we choose $C_{1}=\beta_{1} \gamma_{n}, C_{2}=\beta_{2}$.

Then

$$
K_{n}(z)=\beta_{1} \gamma_{n} \cos \gamma_{n} z+\beta_{2} \sin \gamma_{n} z ; n=1,2, \ldots
$$

and

$$
\widetilde{K}_{n}(z)=\frac{1}{c_{n}} K_{n}(z), \quad c_{n}=\int_{0}^{\ell} K_{n}^{2}(z) d z,
$$


are the kernel of transform with respect to $z$. This transformation is integral transform Fourier. We apply Fourier transform by kernel $\widetilde{K}_{n}(z)$ for $(1)$, and with respect to Fourier representation, we get on the following equation:

$$
\rho c \frac{\partial}{\partial t} \widetilde{T}_{0, n}(r, t)=\frac{\partial}{\partial r}\left(k(r) \frac{\partial}{\partial r} \widetilde{T}_{0, n}\right)-\gamma_{n}^{2} \widetilde{T}_{0, n}+\widetilde{T}_{n}^{*}(r, t), \quad n=1,2, \ldots
$$

where

$$
\begin{aligned}
& \widetilde{T}_{0, n}(r, t)=\int_{0}^{\ell} T_{0}(r, z, t) \widetilde{K}_{n}(z) d z \\
& \widetilde{T}_{n}^{*}(r, t)= {\left[\frac{\partial}{\partial r}\left(k(r) \frac{\partial}{\partial r} T_{1}(r, t)\right)-\rho c \frac{\partial}{\partial t} T_{1}(r, t)\right] \int_{0}^{\ell} \widetilde{K}_{n}(z) d z } \\
&+\left[\frac{\partial}{\partial r}\left(k(r) \frac{\partial}{\partial r} T_{2}(r, t)\right)-\rho c \frac{\partial}{\partial t} T_{2}(r, t)\right] \int_{0}^{\ell} z \widetilde{K}_{n}(z) d z .
\end{aligned}
$$

For $\widetilde{T}_{0, n}(r, t)$ we find initial and boundary conditions as follows:

$$
\begin{aligned}
& \widetilde{T}_{0, n}(r, 0)=\widetilde{\varphi}_{n}(r)-T_{1}(r, 0) \int_{0}^{\ell} \widetilde{K}_{n}(z) d z-T_{2}(r, 0) \int_{0}^{\ell} z \widetilde{K}_{n}(z) d z \\
& {\left.\left[\alpha_{1} \frac{\partial}{\partial r}+\alpha_{2}\right] \widetilde{T}_{0, n}(r, t)\right|_{r=a} }=\widetilde{f}_{n}(t)-\left.\left[\alpha_{1} \frac{\partial}{\partial r}+\alpha_{2}\right] T_{1}(r, t)\right|_{r=a} \int_{0}^{\ell} \widetilde{K}_{n}(z) d z \\
&-\left.\left[\alpha_{1} \frac{\partial}{\partial r}+\alpha_{2}\right] T_{2}(r, t)\right|_{r=a} \int_{0}^{\ell} z \widetilde{K}_{n}(z) d z \equiv \widetilde{f}_{0, n}(t)
\end{aligned}
$$

where

$$
\begin{aligned}
& \widetilde{\varphi}_{n}(r)=\int_{0}^{\ell} \varphi(r, z) \widetilde{K}_{n}(z) d z \\
& \tilde{f}_{n}(t)=\int_{0}^{\ell} f(z, t) \widetilde{K}_{n}(z) d z .
\end{aligned}
$$

In order to be converges uniformly with respect to $r$, we write the solution (16)-(18) on the following formula:

$$
\widetilde{T}_{0, n}(r, t)=\widetilde{T}_{0, n}^{*}(r, t)+\frac{1}{\alpha_{2}} \widetilde{f}_{n}(t),
$$


thus $\widetilde{T}_{0, n}^{*}(r, t)$ satisfies boundary condition:

$$
\left.\left[\alpha_{1} \frac{\partial}{\partial r}+\alpha_{2}\right] \widetilde{T}_{0, n}^{*}(r, t)\right|_{r=a}=\underbrace{\left.\left[\alpha_{1} \frac{\partial}{\partial r}+\alpha_{2}\right] \widetilde{T}_{0, n}(r, t)\right|_{r=a}}_{\widetilde{f}_{0, n}(t)}-\alpha_{2} \cdot \frac{1}{\alpha_{2}} \widetilde{f}_{0, n}(t)=0 .
$$

With respect to $\widetilde{T}_{0, n}^{*}(r, t)$ we get the following equation:

$$
\begin{gathered}
\rho c \frac{\partial}{\partial t} \widetilde{T}_{0, n}^{*}(r, t)=\frac{\partial}{\partial r}\left(k(r) \frac{\partial}{\partial r} \widetilde{T}_{0, n}^{*}(r, t)\right)-\gamma_{n}^{2} \widetilde{T}_{0, n}^{*}(r, t)+\widetilde{T}_{n}^{*}(r, t) \\
-\frac{1}{\alpha_{2}}\left[\rho c \frac{d}{d t}+\gamma_{n}^{2}\right] \widetilde{f}_{0, n}(t), \\
\widetilde{T}_{0, n}^{*}(r, 0)=\widetilde{\varphi}_{0, n}^{*}(r) \\
\left.\widetilde{T}_{0, n}^{*}(r, t)\right|_{r=0}=\left.\widetilde{T}_{0, n}(r, t)\right|_{r=0}-\frac{1}{\alpha_{2}} \widetilde{f}_{0, n}(t)<\infty \\
{\left.\left[\alpha_{1} \frac{\partial}{\partial r}+\alpha_{2}\right] \widetilde{T}_{0, n}^{*}(r, t)\right|_{r=a}=0}
\end{gathered}
$$

where

$$
\widetilde{\varphi}_{0, n}^{*}(r)=\widetilde{\varphi}_{n}(r)-T_{1}(r, 0) \int_{0}^{\ell} \widetilde{K}_{n}(z) d z-T_{2}(r, 0) \int_{0}^{\ell} z \widetilde{K}_{n}(z) d z .
$$

For problem (19)-(22), we use integral transform with respect to $r$.

So that the kernel and weight of this integral be found by using eigenfunctions for Sturm-Liouvill problem following:

$$
\begin{gathered}
\left(k(r) Q^{\prime}(r)\right)^{\prime}+\lambda^{2} Q(r)=0, \quad 0<r<a, \\
Q(0)<\infty,\left.\quad\left[\alpha_{1} \frac{d}{d r}+\alpha_{2}\right] Q(r)\right|_{r=a}=0,
\end{gathered}
$$

the solution of this problem (23) and (24) are the kernel of integral transform with respect to $r$.

Using permutation variables:

$$
r \rightarrow y: \quad y=\frac{2}{2-m} r^{\frac{2-m}{2}} ; r=\left(\frac{2-m}{2} y\right)^{\frac{2}{2-m}},
$$

and when the equation (23) takes the following form 


$$
\frac{\partial^{2}}{\partial y^{2}} Q+\frac{m}{y} \frac{\partial}{\partial y} Q+\left(\frac{\lambda}{\sqrt{k_{0}}}\right)^{2} Q=0,0<r<a .
$$

The solution that bounded when $r=0$, appointed in the following form:

$$
J_{\nu}\left(\frac{\lambda}{\beta \sqrt{k_{0}}} r^{\beta}\right) \cdot r^{(1-m) / 2} ; \beta=\frac{2-m}{2} ; \nu=(1-m) /(2-m) .
$$

Using a second condition of (24), we get

$$
\frac{\alpha_{1}}{\sqrt{k_{0}}} \lambda J_{\nu}^{\prime}\left(\frac{\lambda}{\beta \sqrt{k_{0}}} a^{\beta}\right)+a^{\frac{m}{2}-1}\left((1-m) \frac{\alpha_{1}}{2}+a \alpha_{2}\right) J_{\nu}\left(\frac{\lambda}{\beta \sqrt{k_{0}}} a^{\beta}\right)=0 .
$$

The roots of (25) are all real, simple and have an arithmetic numbers, $\lambda_{\eta}, \eta=1,2, \ldots$ are the roots of the eigenvalues for Sturm-Liouville problem (23) and (24).

Using eigenfunctions corresponding, we find kernel of integral transform with respect to $r$.

$$
K_{\eta}^{*}(r)=\frac{1}{N_{\eta}} J_{\nu}\left(\frac{\lambda_{\eta}}{\beta \sqrt{k_{0}}} r^{\beta}\right) r^{(1-m) / 2}
$$

where

$$
N_{\eta}=\int_{0}^{a}\left(J_{\nu}\left(\frac{\lambda_{\eta}}{\beta \sqrt{k_{0}}} r^{\beta}\right) r^{(1-m) / 2}\right)^{2} d r .
$$

Now using integeral transform with respect to $r$,by kernel $K_{\eta}^{*}(r)$ and weight 1 , we get on problem for representation the function $\widetilde{T}_{0, n}^{*}(r, t)$

$$
\begin{gathered}
\rho c \frac{d}{d t} \widetilde{T}_{0, n, \eta}^{*}(t)=-\mu_{n, \eta}^{2} \widetilde{T}_{0, n, \eta}^{*}(t)+\widetilde{T}_{n, \eta}^{*}(t)-\widetilde{F}_{0, n, \eta}(t), \\
\left.\widetilde{T}_{0, n, \eta}^{*}(t)\right|_{t=0}=\widetilde{\varphi}_{0, n, \eta}^{*},
\end{gathered}
$$

where

$$
\begin{aligned}
\mu_{n, \eta}^{2} & =\left(\lambda_{\eta}^{2}+\gamma_{n}^{2}\right) \\
\widetilde{T}_{n, \eta}^{*}(t) & =\int_{0}^{a} \widetilde{T}_{n}^{*}(r, t) K_{\eta}^{*}(r) d r \\
\widetilde{F}_{0, n, \eta}(t) & =\frac{1}{\alpha_{2}}\left[\rho c \frac{d}{d t}+\gamma_{n}^{2}\right] \widetilde{f}_{0, n}(t) \cdot \int_{0}^{a} K_{\eta}^{*}(r) d r
\end{aligned}
$$




$$
\widetilde{\varphi}_{0, n, \eta}^{*}=\int_{0}^{a} \widetilde{\varphi}_{0, n}^{*}(r) K_{\eta}^{*}(r) d r .
$$

The solution of (26) and (27) as:

$$
\widetilde{T}_{0, n, \eta}^{*}(t)=e^{-\mu_{n, \eta}^{2} /(\rho c) \cdot t}\left[\widetilde{\varphi}_{0, n, \eta}^{*}+\frac{1}{\rho c} \int_{0}^{t}\left(\widetilde{T}_{n, \eta}^{*}(\tau)-\widetilde{F}_{0, n, \eta}(\tau)\right) e^{\mu_{n, \eta}^{2} /(\rho c) \cdot \tau} d \tau\right] .
$$

Using the inverse Hankel transform with respect to $r$, we obtain:

$$
\begin{aligned}
\widetilde{T}_{0, n}^{*}(r, t)= & \sum_{\eta=1}^{\infty} e^{-\mu_{n, \eta}^{2} /(\rho c) \cdot t}\left[\widetilde{\varphi}_{0, n, \eta}^{*}+\frac{1}{\rho c} \int_{0}^{t}\left(\widetilde{T}_{n, \eta}^{*}(\tau)-\widetilde{F}_{0, n, \eta}(\tau)\right)\right. \\
& \left.\cdot e^{\mu_{n, \eta}^{2} /(\rho c) \cdot \tau} d \tau\right] J_{\nu}\left(\frac{\lambda_{\eta}}{\beta \sqrt{k_{0}}} r^{\beta}\right) r^{(1-m) / 2} .
\end{aligned}
$$

Then

$$
\begin{aligned}
\widetilde{T}_{0, n}(r, t)= & \frac{1}{\alpha_{2}} \widetilde{f}_{0, n}(t)+\sum_{\eta=1}^{\infty} e^{-\mu_{n, \eta}^{2} /(\rho c) \cdot t}\left[\widetilde{\varphi}_{0, n, \eta}^{*}+\frac{1}{\rho c} \int_{0}^{t}\left(\widetilde{T}_{n, \eta}^{*}(\tau)\right.\right. \\
& \left.\left.-\widetilde{F}_{0, n, \eta}(\tau)\right) e^{\mu_{n, \eta}^{2} /(\rho c) \cdot \tau} d \tau\right] J_{\nu}\left(\frac{\lambda_{\eta}}{\beta \sqrt{k_{0}}} r^{\beta}\right) r^{(1-m) / 2} .
\end{aligned}
$$

Finally, applying the inverse Fourier transform with respect to $z$, we find the relation to $T(r, z, t)$ :

$$
\begin{aligned}
T(r, z, t)= & T_{1}(r, t)+z T_{2}(r, t)+\sum_{n=1}^{\infty}\left\{\frac{1}{\alpha_{2}} \widetilde{f}_{0, n}(t)+\sum_{\eta=1}^{\infty} e^{-\mu_{n, \eta}^{2} /(\rho c) \cdot t}\right. \\
& \cdot\left[\widetilde{\varphi}_{0, n, \eta}^{*}+\frac{1}{\rho c} \int_{0}^{t}\left(\widetilde{T}_{n, \eta}^{*}(\tau)-\widetilde{F}_{0, n, \eta}(\tau)\right) e^{\mu_{n, \eta}^{2} /(\rho c) \cdot \tau} d \tau\right] \\
& \left.J_{\nu}\left(\frac{\lambda_{\eta}}{\beta \sqrt{k_{0}}} r^{\beta}\right) r^{(1-m) / 2}\right\} K_{n}(z) .
\end{aligned}
$$

\section{Steps Solution using Program Maple 18}

To consider that the outer surface of cylinder is silver: $k_{0}=1, \rho=10.49$, $c=0.0556$, (using the units are c.g.s, caloric, and ${ }^{0} c$ ). 
Assignment of system parameters:

$[>$ restart;

$$
\begin{aligned}
& >S:=\text { unapply }\left(\left(t^{2}+2 t\right), t\right): S(t) \\
& t^{2}+2 t \\
& {\left[>\beta_{1}:=1: \beta_{2}:=2: \beta_{3}:=1: \beta_{4}:=3: \ell:=20:\right.} \\
& a:=1: \alpha_{1}:=1: \alpha_{2}:=1: k_{0}:=1: m:=1: \rho:=10.49: c:=0.0556: \rho \cdot c: \\
& {\left[>g 0:=\text { unapply }\left((1-r)^{2} \cdot t \cdot \mathrm{e}^{-t}, r, t\right): g 0(r, t)\right. \text {; }} \\
& >g l:=\text { unapply }\left((1-r)^{2} \cdot t \cdot \mathrm{e}^{-2 t}, r, t\right): g 1(r, t) \text {; } \\
& \quad(1-r)^{2} t \mathrm{e}^{-2 t} \\
& >f 0:=\operatorname{unapply}\left(1 / \ell \cdot z^{2} \cdot t \cdot \mathrm{e}^{-4 t}, z, t\right): f 0(z, t) \text {; } \\
& \frac{1}{20} z^{2} t \mathrm{e}^{-4 t} \\
& {\left[>f 1:=\text { unapply }\left(1 / \ell \cdot(\ell-z)^{2} \cdot t \cdot \mathrm{e}^{-4 t}, z, t\right): f 1(z, t)\right. \text {; }} \\
& \frac{1}{20}(20-z)^{2} t \mathrm{e}^{-4 t}
\end{aligned}
$$

Representing equation (15) as:

$>\operatorname{tg} \ell:=\operatorname{unapply}\left(\tan (\gamma \cdot \ell) \cdot\left(\gamma^{2} \beta_{1} \beta_{3}-\beta_{2} \beta_{4}\right)-\gamma\left(\beta_{1} \beta_{4}+\beta_{2} \beta_{3}\right), \gamma\right): \operatorname{tg} \ell(\gamma)$

\section{Graph of equation (15):}

$$
\tan (20 \gamma)\left(\gamma^{2}-6\right)-\gamma(5)
$$

$>\operatorname{plot}(\operatorname{tg} \ell(\gamma), \gamma=0 . .1)$;

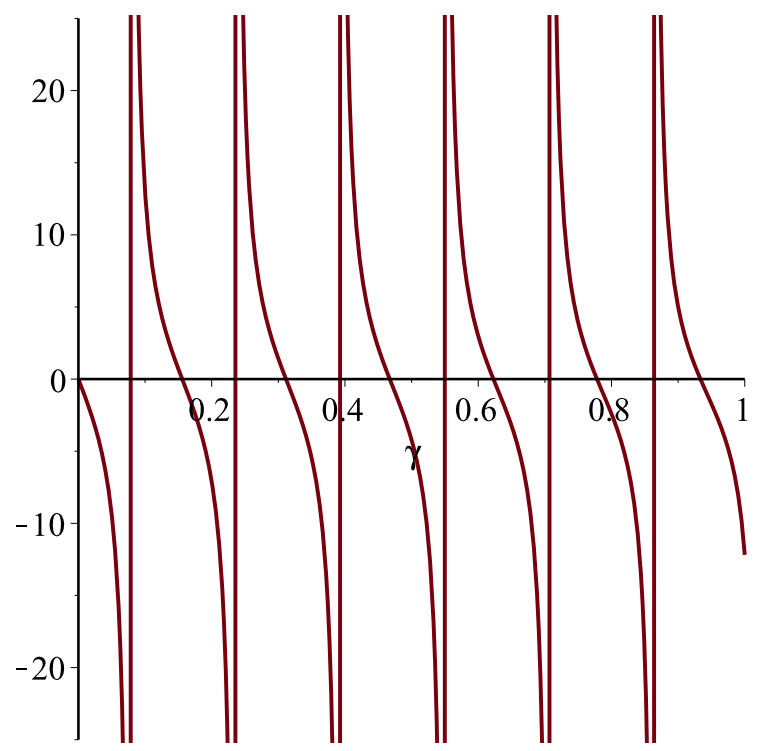

Figuer(1) The roots of the eigenvalue equation.

Evaluation the roots of the eigenvalues equation yields:

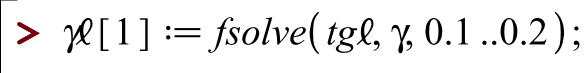




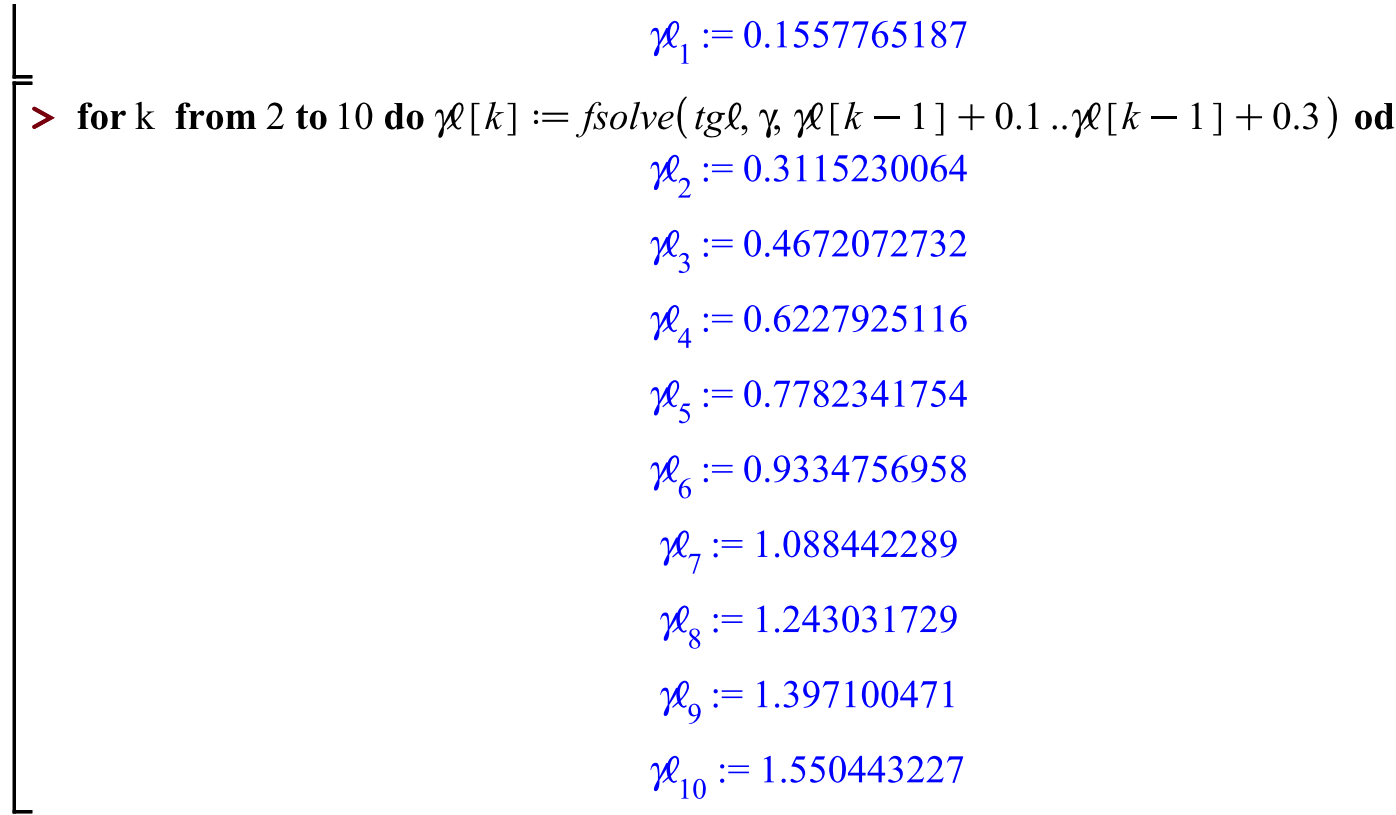

Eigenfunction is given as :

$\left[>K:=\right.$ unapply $\left(\beta_{1} \cdot \mathscr{X}[n] \cdot \cos (\not \mathscr{X}[n] z)+\beta_{2} \cdot \sin (\not \mathscr{X}[n] z), n, z\right): K(n, z):$

Calculate normalized coefficients :

$\left[>C:=\right.$ unapply $\left(\operatorname{int}\left(K(n, z 1)^{2}, z 1=0 . .20\right), n\right): C(n):$

Writing the kernel of Fourier transformation:

$$
\begin{aligned}
& >K T:=\text { unapply }\left(\frac{1}{\mathrm{C}(\mathrm{n})} \cdot K(n, z), n, z\right): K T(n, z) \text {; } \\
& -2\left(\left(\chi_{n} /\left(-\chi_{n}^{2} \cos \left(20 \chi_{n}\right) \sin \left(20 \chi_{n}\right)+4 \chi_{n} \cos \left(20 \chi_{n}\right)^{2}-20 \chi_{n}^{3}\right.\right.\right. \\
& \left.\left.\left.+4 \cos \left(20 \mathscr{\ell}_{n}\right) \sin \left(20 \mathscr{\ell}_{n}\right)-84 \mathscr{X}_{n}\right)\right) \cdot\left(\mathscr{X}_{n} \cos \left(\mathscr{\chi}_{n} z\right)+2 \sin \left(\mathscr{\chi}_{n} z\right)\right)\right) \\
& >\Delta l:=\beta_{1} \cdot \beta_{4}+\beta_{2} \cdot\left(\beta_{4} \cdot \ell+\beta_{3}\right) \text {; }
\end{aligned}
$$

Writing $T_{1}(r, t), T_{2}(r, t)$ as :

$\left[>T 1:=\right.$ unapply $\left(\frac{1}{\Delta l} *\left(g 0(r, t) \cdot\left(\beta_{4} \cdot \ell+\beta_{3}\right)+\beta_{1} \cdot g l(r, t)\right), r, t\right): T 1(r, t):$

$>T 2:=$ unapply $\left(\frac{1}{\Delta l} *\left(\beta_{2} \cdot g l(r, t)-\beta_{4} \cdot g 0(r, t)\right), r, t\right): T 2(r, t):$

Evaluation the roots of the eigenvalue equation as:

$$
\begin{gathered}
>J D:=\text { unapply }\left(\left.\frac{\partial}{\partial r} \operatorname{BesselJ}\left(0, \frac{2 \lambda}{(2-m)} r^{\frac{2-m}{m}}\right)\right|_{r=1}+\operatorname{BesselJ}\left(0, \frac{2 \lambda}{(2-m)} r^{\frac{2-m}{m}}\right)\right. \\
\mid r=1, \lambda): J D(\lambda) ; \\
-2 \operatorname{BesselJ}(1,2 \lambda) \lambda+\operatorname{BesselJ}(0,2 \lambda)
\end{gathered}
$$

Drawing the roots of the eigenvalue equation to be given as:

$\lceil\operatorname{plot}(J D(\lambda), \lambda=0 . .10)$; 


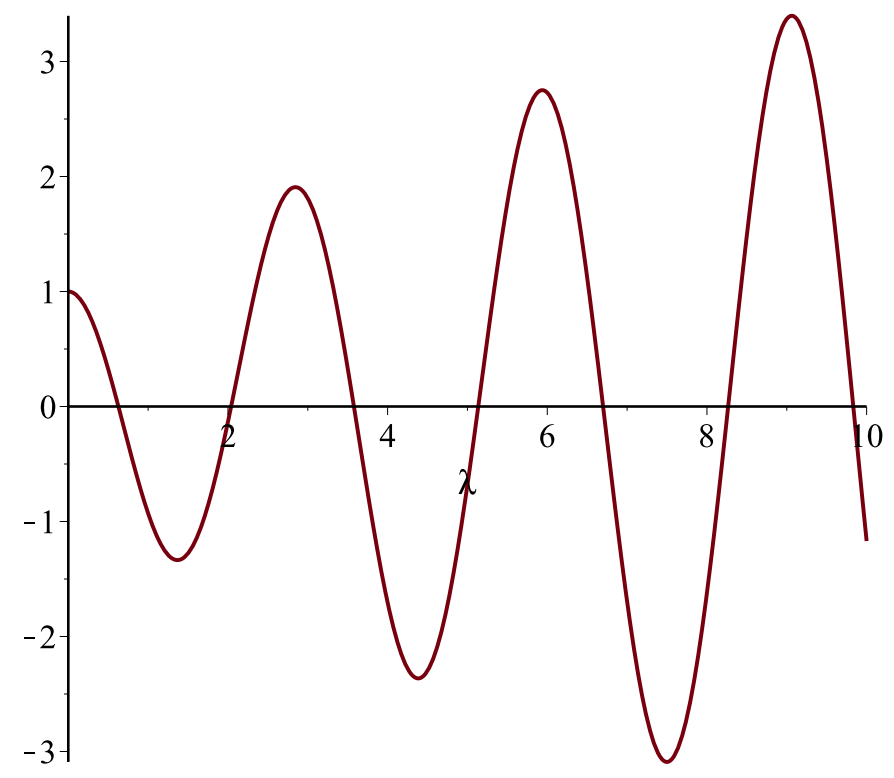

Figuer(2) The roots of the eigenvalue equation.

Evaluation the roots of the eigenvalues equation yields:

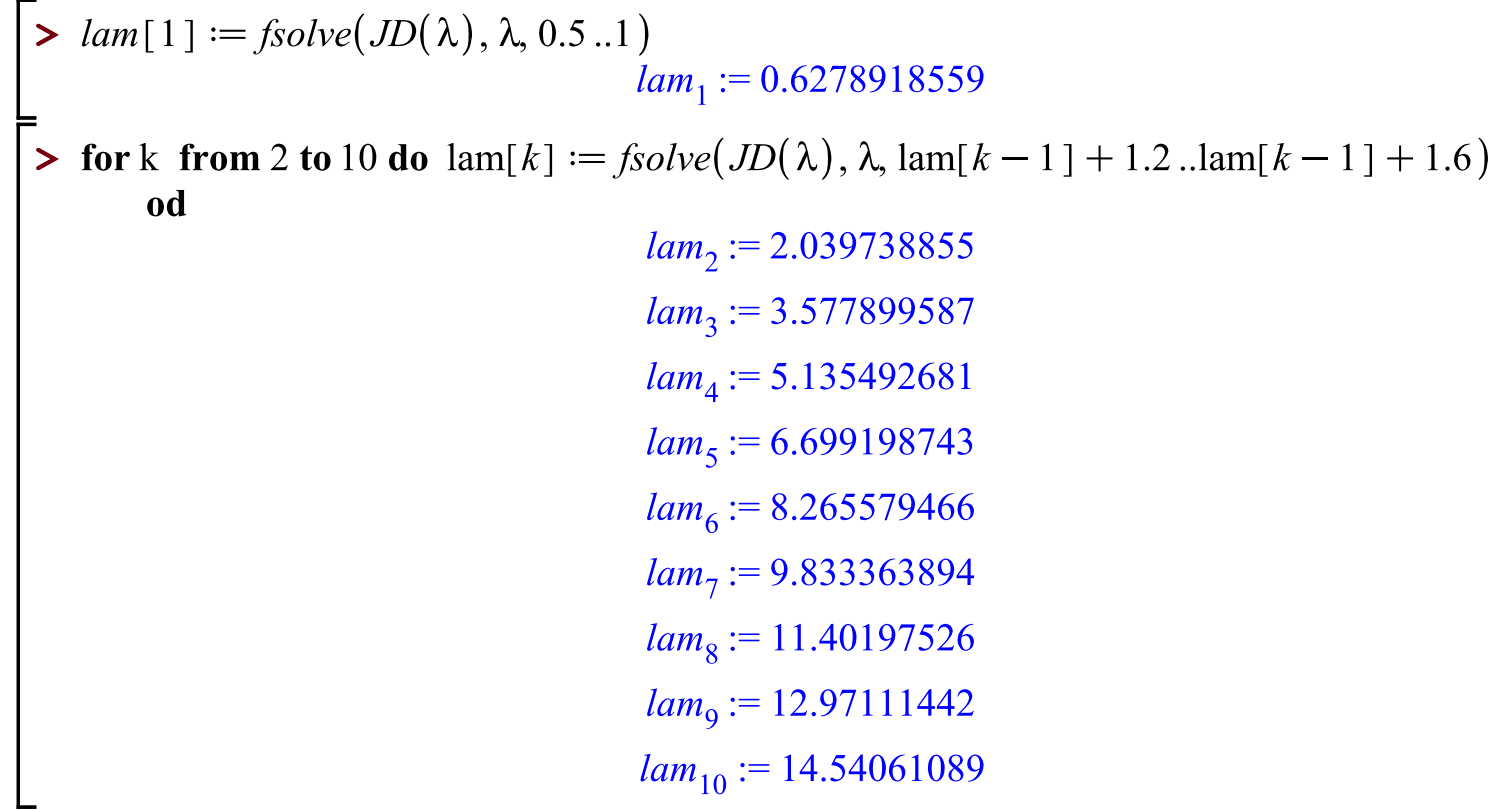

Insertion of the initial function as:

$$
\left[\begin{array}{r}
>\varphi:=\text { unapply }\left(\frac{1}{a \cdot \ell} r \cdot(1-r)^{2} \cdot z^{2} \cdot(\ell-z)^{2}, r, z\right): \varphi(r, z) \\
\frac{1}{20} r(1-r)^{2} z^{2}(20-z)^{2}
\end{array}\right.
$$

Insertion of the assistant operators as:

$\left[>I F 1:=\operatorname{unapply}\left(\operatorname{int}\left(f 1(z 1, t) K T(n, z 1), z 1=0 . .\left(t^{2}+2 t\right)\right), n, t\right): \operatorname{IF1}(n, t):\right.$

$\left[>\operatorname{IF0}:=\operatorname{unapply}\left(\operatorname{int}\left(f 0(z 1, t) K T(n, z 1), z 1=\left(t^{2}+2 t\right) . . \ell\right), n, t\right): \operatorname{IF0}(n, t):\right.$ 


$$
\begin{aligned}
& \mid>\operatorname{IF01}:=\text { unapply }(\operatorname{IFO}(n, t)+\operatorname{IF} 1(n, t), n, t): \operatorname{IF01}(n, t) ;
\end{aligned}
$$

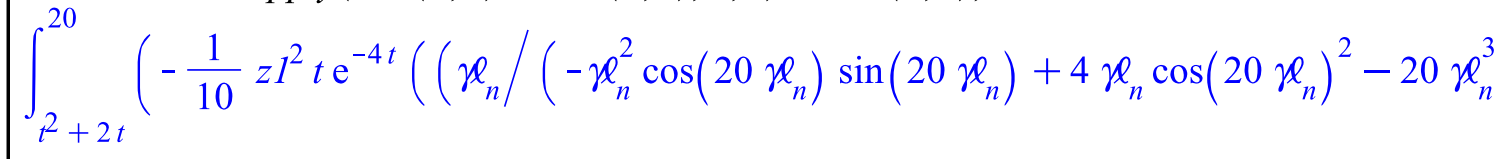

$$
\begin{aligned}
& \left.\left.\left.\left.+4 \cos \left(20 \mathscr{X}_{n}\right) \sin \left(20 \mathscr{\chi}_{n}\right)-84 \mathscr{\chi}_{n}\right)\right) \cdot\left(\mathscr{\chi}_{n} \cos \left(\mathscr{\chi}_{n} z 1\right)+2 \sin \left(\mathscr{X}_{n} z 1\right)\right)\right)\right) \mathrm{d} z 1+ \\
& \int_{0}^{t^{2}+2 t}\left(-\frac{1}{10}(20-z 1)^{2} t \mathrm{e}^{-4 t}\left(\left(\not_{n} /\left(-\not \chi_{n}^{2} \cos \left(20 \not_{n}\right) \sin \left(20 \mathscr{\chi}_{n}\right)\right.\right.\right.\right. \\
& \left.\left.+4 \mathscr{X}_{n} \cos \left(20 \mathscr{X}_{n}\right)^{2}-20 \not \mathscr{X}_{n}^{3}+4 \cos \left(20 \mathscr{X}_{n}\right) \sin \left(20 \mathscr{X}_{n}\right)-84 \mathscr{\chi}_{n}\right)\right) \cdot\left(\mathscr{\chi}_{n} \cos \left(\mathscr{\chi}_{n} z 1\right)\right. \\
& \left.\left.\left.+2 \sin \left(\chi_{n} z l\right)\right)\right)\right) \mathrm{d} z l
\end{aligned}
$$

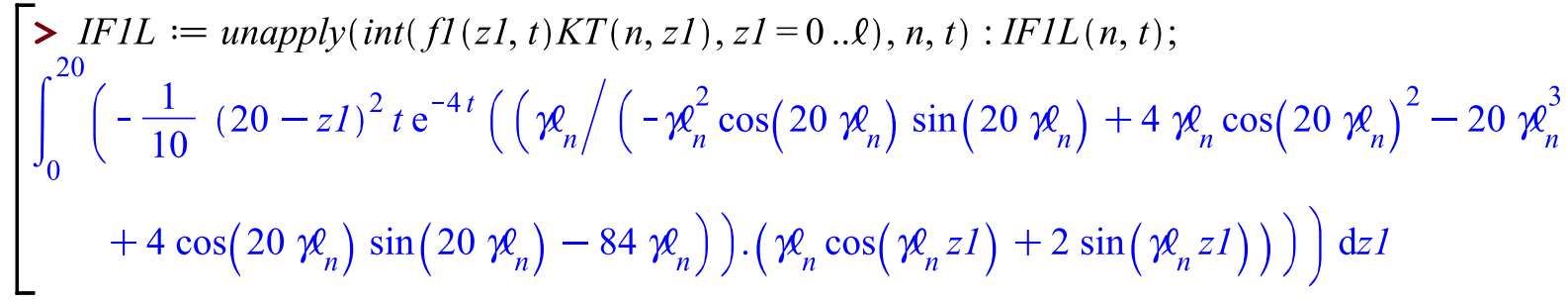

Calculate normalized coefficients :

$\left[>N:=\right.$ unapply $\left(\operatorname{int}\left(\operatorname{BesselJ}\left(0, \frac{2 \operatorname{lam}[\eta]}{(2-m)} r^{\frac{2-m}{2}}\right)^{2}, r=0 . . a\right), \eta\right): N(\eta):$

Writing the kernel of Hankel transformation:

$\left[>K S:=\right.$ unapply $\left(\frac{1}{N(\eta)} \cdot \operatorname{BesselJ}\left(0, \frac{2 \operatorname{lam}[\eta]}{(2-m)} r^{\frac{2-m}{2}}\right), \eta, r\right): K S(\eta, r):$

Taking $\mu$ the following formula:

$$
\begin{aligned}
& {\left[>\mu:=\text { unapply }\left(\frac{\left(\operatorname{lam}[\eta]^{2}+\not \mathcal{X}[n]^{2}\right)}{\rho \cdot c}, n, \eta\right): \mu(n, \eta)\right. \text {; }} \\
& 1.714548285 \operatorname{lam}_{\eta}^{2}+1.714548285 \not \mathcal{X}_{n}^{2}
\end{aligned}
$$

Assign two integral of the (IK-IKZ):

$$
\begin{aligned}
& {[>I K:=\text { unapply }(\operatorname{int}(K T(n, z 1), z 1=0 . . \ell), n): I K(n):} \\
& {[>I K Z:=\text { unapply }(\operatorname{int}(z 1 \cdot K T(n, z 1), z 1=0 . . \ell), n): I K Z(n):} \\
& {\left[>R 1:=\text { unapply }\left(\left.\alpha_{1} \frac{\partial}{\partial r} T 1(r, t)\right|_{r=a}+\left.\alpha_{2} T 1(r, t)\right|_{r=a}, t\right): R 1(t):\right.} \\
& {\left[>R 2:=\text { unapply }\left(\left.\alpha_{1} \frac{\partial}{\partial r} T 2(r, t)\right|_{r=a}+\left.\alpha_{2} T 2(r, t)\right|_{r=a}, t\right): R 2(t):\right.} \\
& {[>R 11:=\text { unapply }(R 1(t) I K(n), n, t): R 11(n, t):} \\
& {[>R 22:=\text { unapply }(R 2(t) I K Z(n), n, t): R 22(n, t):} \\
& {[>R 11 R 22:=\text { unapply }(R 11(n, t)+R 22(n, t), n, t): R 11 R 22(n, t):}
\end{aligned}
$$


Suppose that two case:

\section{Case1:}

when $t<\sqrt{\ell+1}-1$, if the outer surface of the entire cylinder under the effect two waves $f 1(z, t), f 0$ $(z, t)$, thus the function takes on the IF01:

$$
\begin{aligned}
& {\left[\begin{array}{l}
>\text { FON1S := unapply }(\operatorname{IFO} 01(n, t)-R 11 R 22(n, t), n, t): \text { F0N1S }(n, t) ; \\
\int_{t^{2}+2 t}^{20}\left(-\frac{1}{10} z l^{2} t \mathrm{e}^{-4 t}\left(\left(\mathcal{X}_{n} /\left(-\mathcal{X}_{n}^{2} \cos \left(20 \mathcal{X}_{n}\right) \sin \left(20 \mathcal{X}_{n}\right)+4 \mathcal{X}_{n} \cos \left(20 \mathcal{X}_{n}\right)^{2}-20 \mathcal{X}_{n}^{3}\right.\right.\right.\right.
\end{array}\right.} \\
& \left.\left.\left.\left.+4 \cos \left(20 \mathscr{\chi}_{n}\right) \sin \left(20 \mathscr{\chi}_{n}\right)-84 \mathscr{\chi}_{n}\right)\right) \cdot\left(\mathscr{\chi}_{n} \cos \left(\varkappa_{n} z 1\right)+2 \sin \left(\varkappa_{n} z 1\right)\right)\right)\right) \mathrm{d} z 1+ \\
& \int_{0}^{t^{2}+2 t}\left(-\frac{1}{10}(20-z 1)^{2} t \mathrm{e}^{-4 t}\left(\left(\not_{n} /\left(-\chi_{n}^{2} \cos \left(20 \chi_{n}\right) \sin \left(20 \not_{n}\right)\right.\right.\right.\right.
\end{aligned}
$$

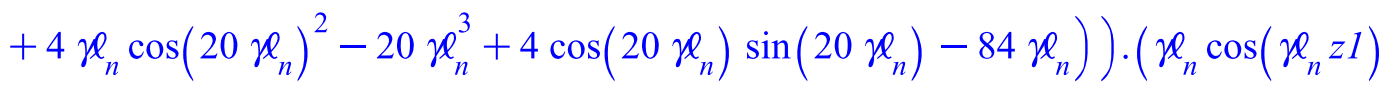

$$
\begin{aligned}
& \left.\left.\left.+2 \sin \left(\mathcal{\ell}_{n} z l\right)\right)\right)\right) \mathrm{d} z 1
\end{aligned}
$$

\section{Case2:}

when $t>\sqrt{\ell+1}-1$, if the outer surface of the entire cylinder under the effect two waves f1 $(z, t)$, thus the function takes on the IFIL:

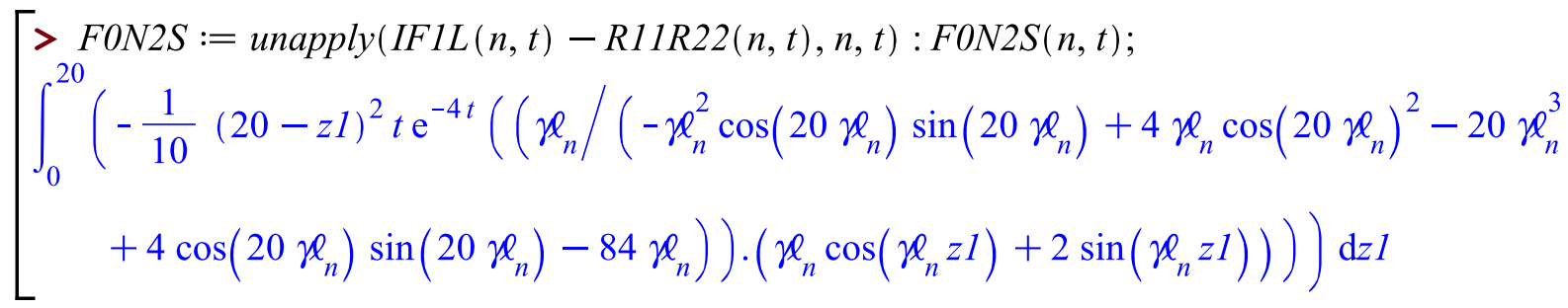

$$
\begin{aligned}
& {[>\varphi T:=\operatorname{unapply}(\operatorname{int}(\varphi(r, z l) K T(n, z l), z 1=0 . . \ell), n, r): \varphi T(n, r):} \\
& {[>\varphi 0 T S:=\text { unapply }((\varphi T(n, r)-T 1(r, 0) . I K(n)-T 2(r, 0) . I K Z(n)), n, r): \varphi 0 T S(n, r):} \\
& >\varphi 0 T S T:=\text { unapply }(\operatorname{int}(\varphi 0 T S(n, r l) K S(\eta, r l), r l=0 . . a), n, \eta): \varphi 0 T S T(n, \eta) \text {; } \\
& \int_{0}^{1}\left(\int _ { 0 } ^ { 2 0 } \left(-\frac{1}{10} r l(1-r l)^{2} z l^{2}(20-z l)^{2}\left(\left(\varkappa_{n} /\left(-\not \ell_{n}^{2} \cos \left(20 \mathscr{\ell}_{n}\right) \sin \left(20 \not_{n}\right)\right.\right.\right.\right.\right.
\end{aligned}
$$

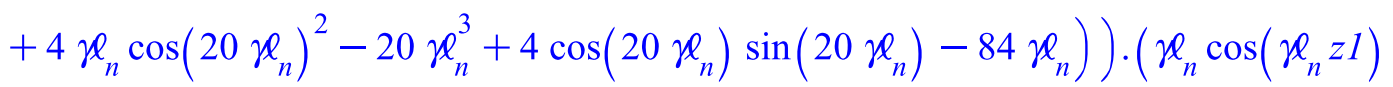

$$
\begin{aligned}
& \left.\left.\left.\left.+2 \sin \left(\not \mathscr{L}_{n} z l\right)\right)\right)\right) \mathrm{d} z l\right)\left(\left(\frac{1}{\operatorname{BesselJ}\left(0,2 \operatorname{lam}_{\eta}\right)^{2}+\operatorname{BesselJ}\left(1,2 \operatorname{lam}_{\eta}\right)^{2}}\right) \cdot \operatorname{BesselJ}(0,\right. \\
& \left.\left.2 \operatorname{lam}_{\eta} \sqrt{r 1}\right)\right) \mathrm{d} r 1 \\
& \bar{\Gamma} \text { TTS }:=\text { unapply }\left(\left(\frac{\partial}{\partial r}\left(k_{0} \cdot r^{m} \cdot \frac{\partial}{\partial r} T 1(r, t)\right)-(\rho \cdot c) \cdot \frac{\partial}{\partial t} T 1(r, t)\right) \cdot I K(n)\right. \\
& \left.+\left(\frac{\partial}{\partial r}\left(k_{0} r^{m} \cdot \frac{\partial}{\partial r} T 2(r, t)\right)-(\rho \cdot c) \cdot \frac{\partial}{\partial t} T 2(r, t)\right) \cdot \operatorname{IKZ}(n), n, r, t\right): \operatorname{TTS}(n, r, t) ; \\
& \left(-\frac{122}{125}(1-r) t \mathrm{e}^{-t}-\frac{2}{125}(1-r) t \mathrm{e}^{-2 t}+r \cdot\left(\frac{122}{125} t \mathrm{e}^{-t}+\frac{2}{125} t \mathrm{e}^{-2 t}\right)-0.2846230720(1\right.
\end{aligned}
$$




$$
\begin{aligned}
& -r)^{2} \mathrm{e}^{-t}+0.2846230720(1-r)^{2} t \mathrm{e}^{-t}-0.004665952000(1-r)^{2} \mathrm{e}^{-2 t}
\end{aligned}
$$

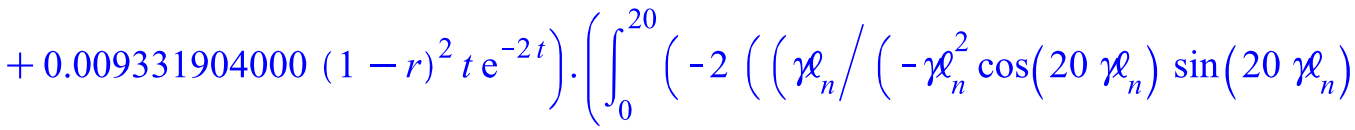

$$
\begin{aligned}
& \left.\left.+4 \varkappa_{n} \cos \left(20 \varkappa_{n}\right)^{2}-20 \chi_{n}^{3}+4 \cos \left(20 \varkappa_{n}\right) \sin \left(20 \varkappa_{n}\right)-84 \varkappa_{n}\right)\right) \cdot\left(\varkappa_{n} \cos \left(\varkappa_{n} z l\right)\right. \\
& \left.\left.\left.\left.+2 \sin \left(\not \mathscr{X}_{n} z 1\right)\right)\right)\right) \mathrm{d} z 1\right)+\left(-\frac{4}{125}(1-r) t \mathrm{e}^{-2 t}+\frac{6}{125}(1-r) t \mathrm{e}^{-t}+r \cdot\left(\frac{4}{125} t \mathrm{e}^{-2 t}\right.\right. \\
& \left.-\frac{6}{125} t \mathrm{e}^{-t}\right)-0.009331904000(1-r)^{2} \mathrm{e}^{-2 t}+0.01866380800(1-r)^{2} t \mathrm{e}^{-2 t} \\
& \left.+0.01399785600(1-r)^{2} \mathrm{e}^{-t}-0.01399785600(1-r)^{2} t \mathrm{e}^{-t}\right) \cdot\left(\int _ { 0 } ^ { 2 0 } \left(-2\left(z 1 \cdot \left(\not_{n} /(-\right.\right.\right.\right.
\end{aligned}
$$

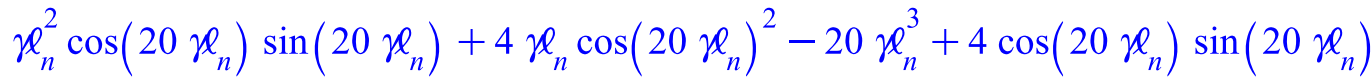

$$
\begin{aligned}
& \left.\left.\left.\left.\left.-84 \mathscr{X}_{n}\right)\right) \cdot\left(\mathscr{\chi}_{n} \cos \left(\mathscr{\chi}_{n} z 1\right)+2 \sin \left(\mathscr{\chi}_{n} z l\right)\right)\right)\right) \mathrm{d} z 1\right)
\end{aligned}
$$

TTSNT $:=\operatorname{unapply}(\operatorname{int}(\operatorname{TTS}(n, r l, t) \cdot K S(\eta, r l), r l=0 . . a), n, \eta, t): \operatorname{TTSNT}(n, \eta, t):$

FTONT $:=$ unapply $\left(\left(\rho \cdot c \frac{\partial}{\partial t} F 0 N 2 S(n, t)+(\not{X}[n])^{2} F 0 N 2 S(n, t)\right) \cdot \operatorname{int}(K S(\eta, r l), r l=0 \ldots a)\right.$, $n, \eta, t): F T O N T(n, \eta, t):$

$[>\operatorname{assume}(t>0)$;

$>\operatorname{ITTSFT}:=\operatorname{unapply}\left(\operatorname{int}\left(\mathrm{e}^{\mu(n, \eta) \cdot \tau}(\operatorname{TTSNT}(n, \eta, \tau)-\operatorname{FTONT}(n, \eta, \tau)), \tau=0 . . t\right), n, \eta, t\right):$

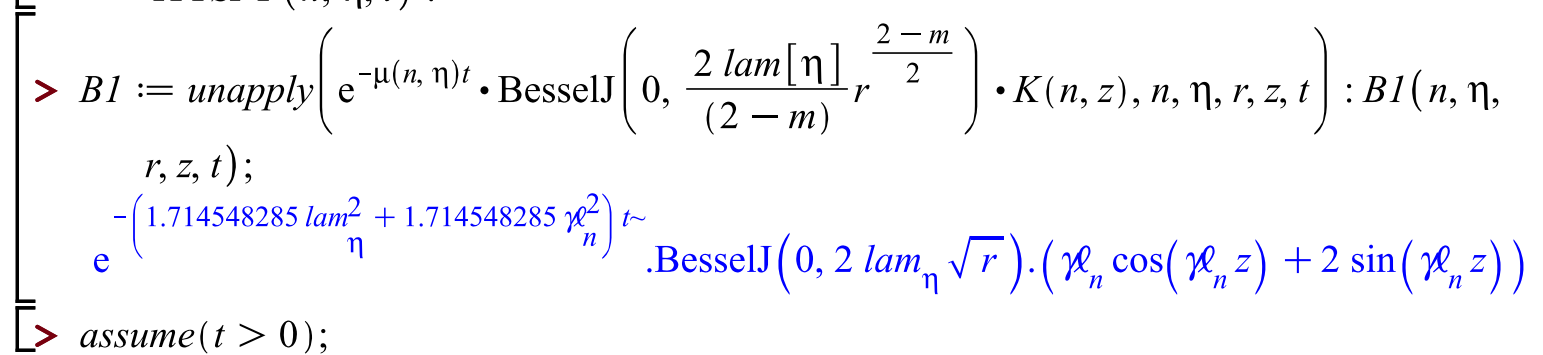

$\left[>B 2:=\right.$ unapply $\left(\left(\frac{1}{\rho \cdot c}\right) \cdot(\operatorname{ITTSFT}(n, \eta, t)+\varphi 0 T S T(n, \eta)), n, \eta, t\right): B 2(n, \eta, t):$

$>T 12:=$ unapply $(T 1(r, t)+z * T 2(r, t), r, z, t): T 12(r, z, t)$;

$$
\begin{aligned}
& \frac{61}{125}(1-r)^{2} t \sim \mathrm{e}^{-t \sim}+\frac{1}{125}(1-r)^{2} t \sim \mathrm{e}^{-2 t \sim}+z\left(\frac{2}{125}(1-r)^{2} t \sim \mathrm{e}^{-2 t \sim}-\frac{3}{125}(1\right. \\
& \left.-r)^{2} t \sim \mathrm{e}^{-t \sim}\right)
\end{aligned}
$$

General solution for case1:

$[>\operatorname{assume}(t>0)$;

$>$ U0 $:=\operatorname{unapply}(\operatorname{T12}(r, z, t)+\operatorname{Sum}(\operatorname{IF01}(n, t) \cdot K(n, z), n=1 . .5)+\operatorname{Sum}(\operatorname{Sum}(B 1(n, \eta, r$, $z, t) \cdot B 2(n, \eta, t), \eta=1 . .5), n=1 . .5), r, z, t): U 0(r, z, t):$

Assingn a plot of the function U0(z) for case1:

$>\operatorname{plot}(U 0(0.1, z, 1), z=3 . .18)$ 


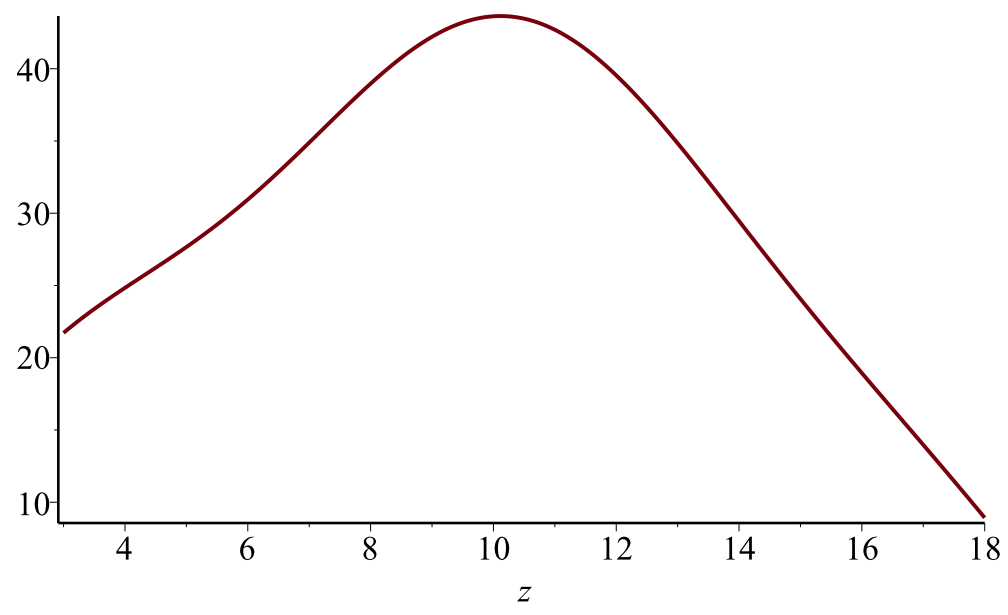

Figure(3)

Figure ( 3) shows the variation of temperature a long the $r$ direction, and it is seen easily from the curve that the temperature satisfies the highest value at $\mathrm{z}=10$ (local maximum), low value at $\mathrm{z}=18$. Assignment graphic of spread heat for circle $z=6$, at time $t=1$ over the interval $r \in[0.1,0.9]$ :

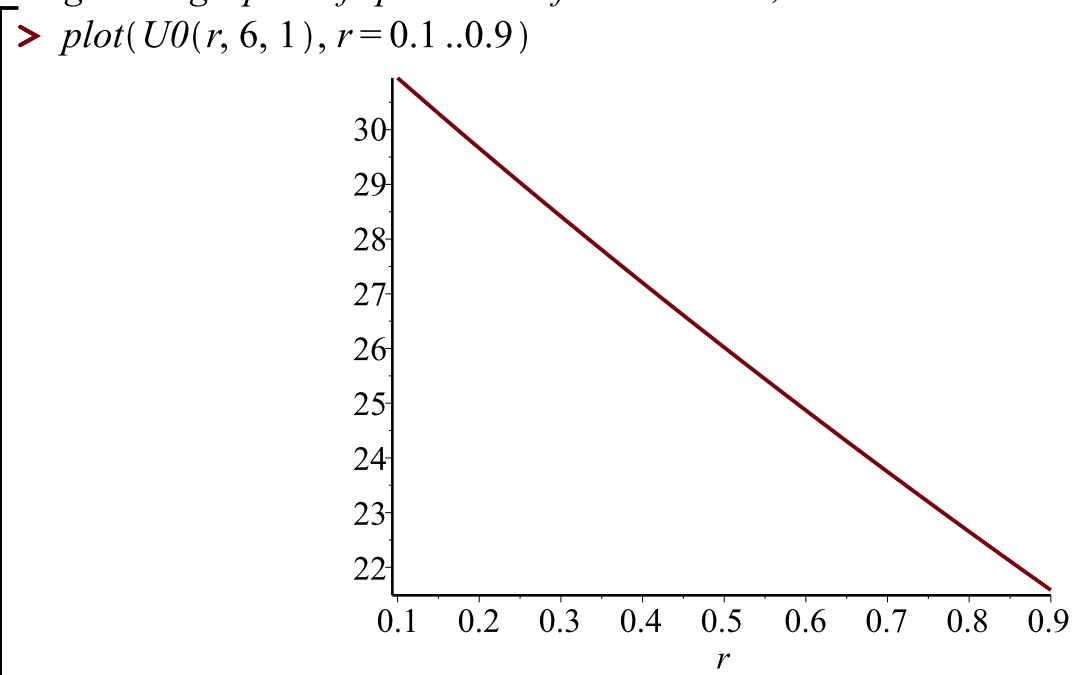

Figure(4)

Figure(4) shows the convex curve, and direction from the top to the bottom also temperatures regularly drop.

$$
\begin{aligned}
& {\left[>\text { addcoords }\left(z \_ \text {cylindrical },[z, r, \theta],[r \cos (\theta), r \sin (\theta), z]\right)\right.} \\
& {\left[\begin{array}{c}
>\operatorname{plot} 3 d\left(U 0(r, 6,1), r=0.1 . .0 .9, \theta=0 . .2 \pi, \text { coords }=z \text { cylindrical, title }=z_{-}\right. \text {cylindrical, } \\
\text { orientation }=[-132,71], \text { axes }=\text { boxed })
\end{array}\right.}
\end{aligned}
$$




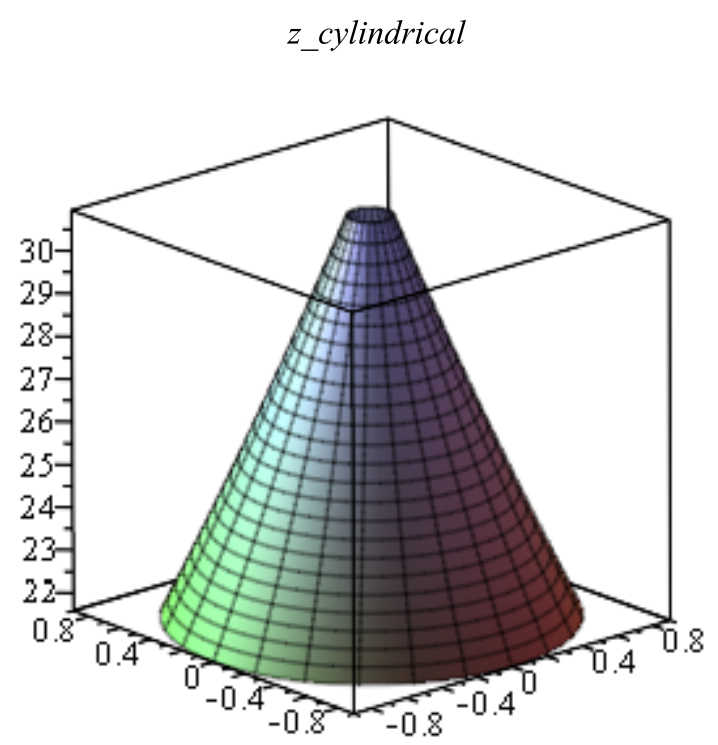

Figure(5) show the spread heat in the cylindrical coordinates.

General solution for case2:

$[>\operatorname{assume}(t>0)$;

$>U 1:=$ unapply $(T 12(r, z, t)+\operatorname{Sum}(\operatorname{IF} 1 L(n, t) \cdot K(n, z), n=1 . .5)+\operatorname{Sum}(\operatorname{Sum}(B 1(n, \eta, r$, $z, t) \cdot B 2(n, \eta, t), \eta=1 . .5), n=1 . .5), r, z, t):$

$>\operatorname{plot} 3 d\left(U 1(r, 6,4), r=0.1 . .0 .9, \theta=0 . .2 \pi\right.$, coords $=z_{-}$cylindrical, title $=z_{-}$cylindrical, orientation $=[-132,71]$, axes $=$ boxed $)$

z_cylindrical

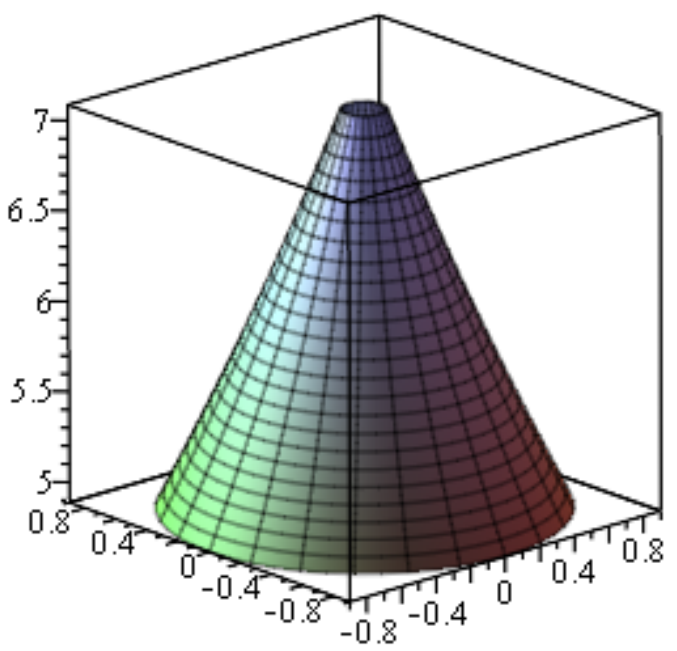


Figure(6) show the spread heat in the cylindrical coordinates.

Assignment graphic of spread heat for circle $z=6$, at time $t=4$ over the interval $r \in[0.1,0.9]$ :

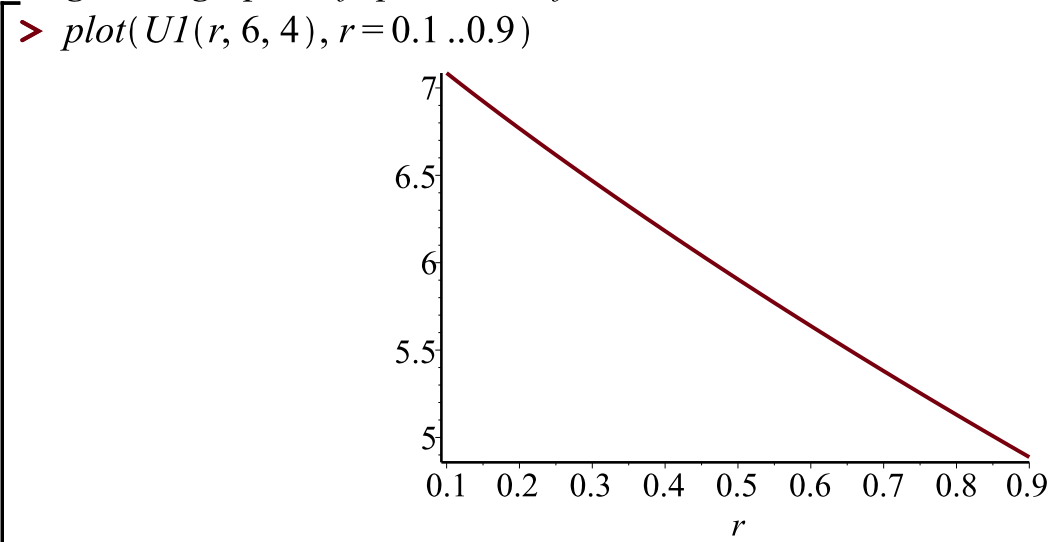

\section{Figure(7)}

Figure(7) it is seen that the drop of temperature from 7.1 into 4.9 clearly,and the convex curve direction into top.

Assingn a plot of the function U1(z) for case 2:

$>\operatorname{plot}(U 1(0.1, z, 4), z=3 . .18)$

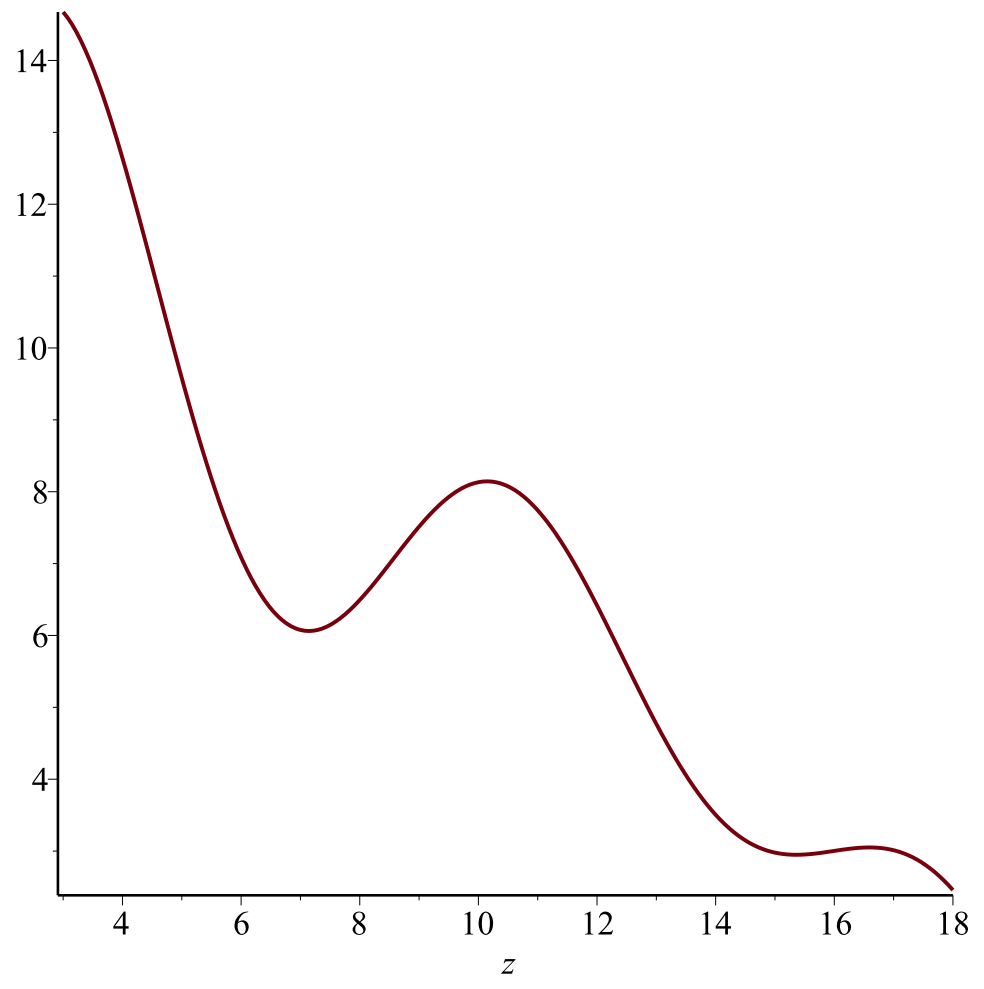

Figure (8) it is evident that the curve one local minimum values at $(\mathrm{z}=7)$, and one local maximum value at $(\mathrm{z}=10.5)$. 
Assignment of tables to spread heat in both cases, while $\mathrm{n} \times \eta=5 \times 5, \mathrm{z} \in[3,18], \mathrm{r} \in[0.1,0.9]$ :

[> $\mathrm{r}:=0: \mathrm{z}:=0: \mathrm{t}:=1:$

$>s:=\operatorname{array}(1 . .6,1 . .9): \mathrm{j}:=0: \mathrm{k}:=0$ :

while $\mathrm{z}<18$ do $\mathrm{z}:=\mathrm{z}+3: \mathrm{k}:=\mathrm{k}+1:$

while $\mathrm{r}<0.9$ do $\mathrm{r}:=\mathrm{r}+0.1: \mathrm{j}:=\mathrm{j}+1: s[\mathrm{k}, \mathrm{j}]:=0$ :

for $n$ from 1 to 5 do for $\eta$ from 1 to 5 do

$b:=\operatorname{evalf}(T 12(r, z, t)+\operatorname{IF01}(n, t) \cdot K(n, z)+B 1(n, \eta, r, z, t) \cdot B 2(n, \eta, t)) ; s[\mathrm{k}, \mathrm{j}]$ $:=s[\mathrm{k}, \mathrm{j}]+b$

od od od; $r:=0 ; j:=0 ;$ od;

for $\mathrm{k} 1$ from 1 to $6 \mathrm{do} \mathrm{z}:=3 \cdot \mathrm{k} 1 ; \operatorname{print}(\mathrm{z})$; for $\mathrm{j} 1$ from 1 to $9 \operatorname{do} \operatorname{print}(s[\mathrm{k} 1, \mathrm{j} 1]) \operatorname{od}$ od ;

\begin{tabular}{|c|c|c|}
\hline$z:=3$ & $z:=6$ & $z:=9$ \\
\hline 25.74899406 & 33.54895660 & 44.99047685 \\
\hline 23.61029626 & 31.68997961 & 42.68268936 \\
\hline 21.88110043 & 29.93746138 & 40.53140884 \\
\hline 20.34710800 & 28.28585092 & 38.49289982 \\
\hline 18.97584904 & 26.73300879 & 36.56024858 \\
\hline 17.76495681 & 25.27768077 & 34.73262251 \\
\hline 16.70422765 & 23.91876611 & 33.00759018 \\
\hline 15.77627011 & 22.65522539 & 31.38125903 \\
\hline 14.96442149 & 21.48613849 & 29.84988394 \\
\hline
\end{tabular}

$\left[\begin{array}{c}z:=12 \\ 41.95421990 \\ 39.87031088 \\ 37.91329253 \\ 36.05177212 \\ 34.28059778 \\ 32.59898723 \\ 31.00505219 \\ 29.49588493 \\ 28.06869432\end{array}\right]\left[\begin{array}{c}z:=15 \\ 26.49869427 \\ 25.24300034 \\ 22.0271564961 .75797497 \\ 20.70582606 \\ 19.70876442 \\ 18.76758455 \\ 17.88306525\end{array}\right]\left[\begin{array}{c}z:=18 \\ 11.16406893 \\ 10.47207286 \\ 9.865530425 \\ 9.310442476 \\ 8.801901760 \\ 7.339726442 \\ 7.5422396286 \\ 7.211461663\end{array}\right]$

$>\mathrm{r}:=0: \mathrm{z}:=0: \mathrm{t}:=4:$

$>s:=\operatorname{array}(1 . .6,1 . .9): \mathrm{j}:=0: \mathrm{k}:=0:$

while $\mathrm{z}<18$ do $\mathrm{z}:=\mathrm{z}+3: \mathrm{k}:=\mathrm{k}+1:$

while $\mathrm{r}<0.9$ do $\mathrm{r}:=\mathrm{r}+0.1: \mathrm{j}:=\mathrm{j}+1: s[\mathrm{k}, \mathrm{j}]:=0$ :

for $n$ from 1 to 5 do for $\eta$ from 1 to 5 do

$b:=\operatorname{evalf}(T 12(r, z, t)+\operatorname{IF1L}(n, t) \cdot K(n, z)+B 1(n, \eta, r, z, t) \cdot B 2(n, \eta, t)) ; s[\mathrm{k}, \mathrm{j}]$ $:=s[\mathrm{k}, \mathrm{j}]+b$

od od od; $r:=0 ; j:=0 ;$ od;

for $\mathrm{k} 1$ from 1 to 6 do $\mathrm{z}:=3 \cdot \mathrm{k} 1 ; \operatorname{print}(\mathrm{z})$; for $\mathrm{j} 1$ from 1 to 9 do $\operatorname{print}(s[\mathrm{k} 1, \mathrm{j} 1])$ od od ; 


\begin{tabular}{|c|c|c|c|c|}
\hline$z:=3$ & $z:=6$ & $z:=9$ & $z:=12$ & $z:=15$ \\
\hline 15.26807468 & 7.578065607 & 7.905372260 & 6.706959739 & 3.166973154 \\
\hline 13.94938115 & 7.157224611 & 7.399421312 & 6.299744498 & 3.042145738 \\
\hline 12.96989124 & 6.766384035 & 6.972434919 & 5.950503627 & 2.905227269 \\
\hline 12.11537293 & 6.400249464 & 6.581051260 & 5.628201256 & 2.770243667 \\
\hline 11.35342264 & 6.056934546 & 6.218724910 & 5.328037394 & 2.638976361 \\
\hline 10.68173934 & 5.735434887 & 5.884986769 & 5.049576904 & 2.511274976 \\
\hline 10.09018487 & 5.434896198 & 5.577763805 & 4.791273908 & 2.387605986 \\
\hline 9.561432870 & 5.154522439 & 5.293517249 & 4.550558541 & 2.268977758 \\
\hline 9.078885078 & 4.893633707 & 5.028851185 & 4.324973221 & 2.156380295 \\
\hline$z:=18$ & & & & \\
\hline 2.544818466 & & & & \\
\hline 2.336787770 & & & & \\
\hline 2.181262220 & & & & \\
\hline 2.044309068 & & & & \\
\hline 1.921086659 & & & & \\
\hline 1.811477840 & & & & \\
\hline 1.714026206 & & & & \\
\hline 1.626070201 & & & & \\
\hline 1.545035925 & & & & \\
\hline
\end{tabular}

Using the precedent table we construct curves of spread heat for $\mathrm{U} 1=\mathrm{U} 1(\mathrm{r})$ of inner layers $(\mathrm{r}=0.1$. .0.9):

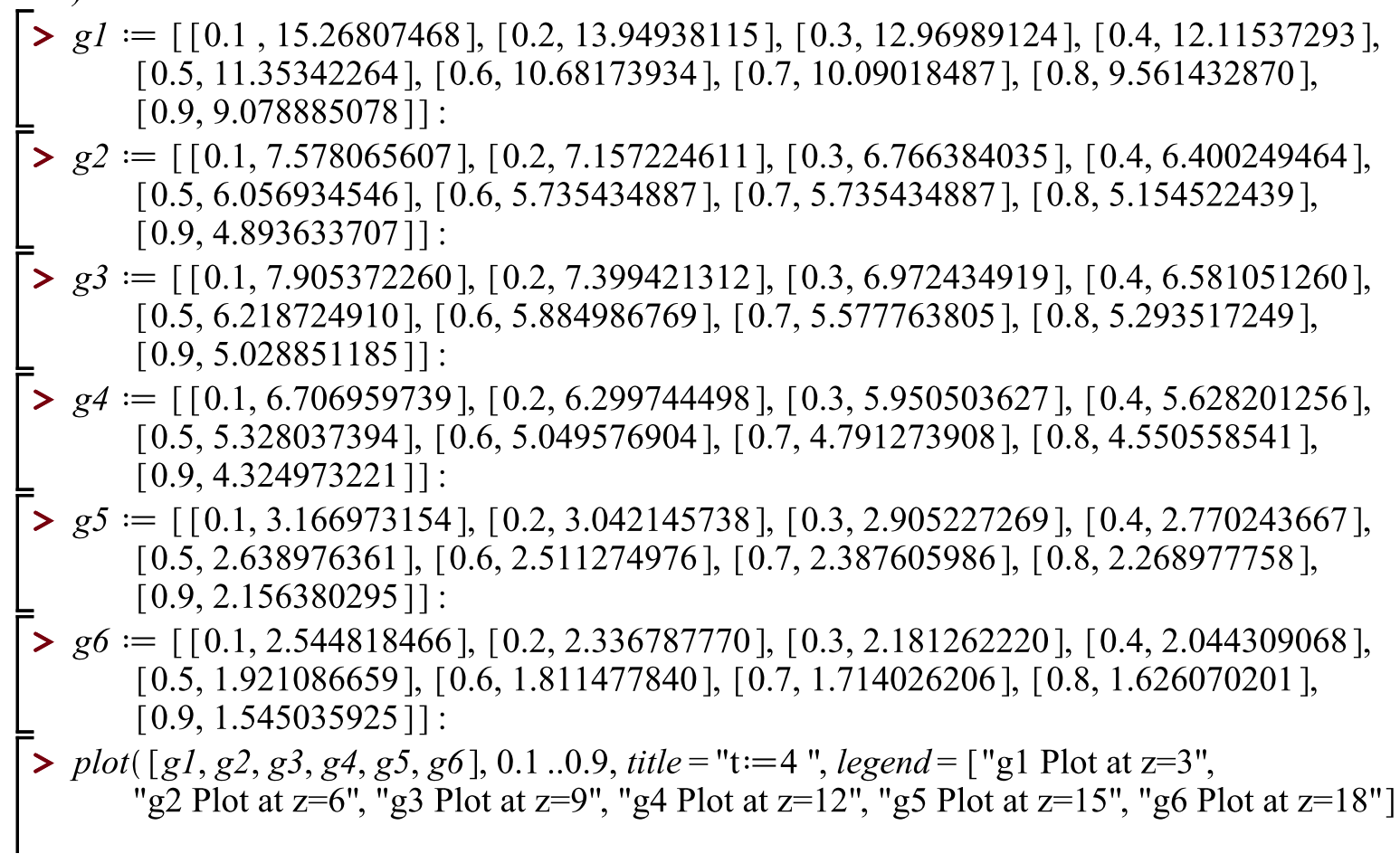




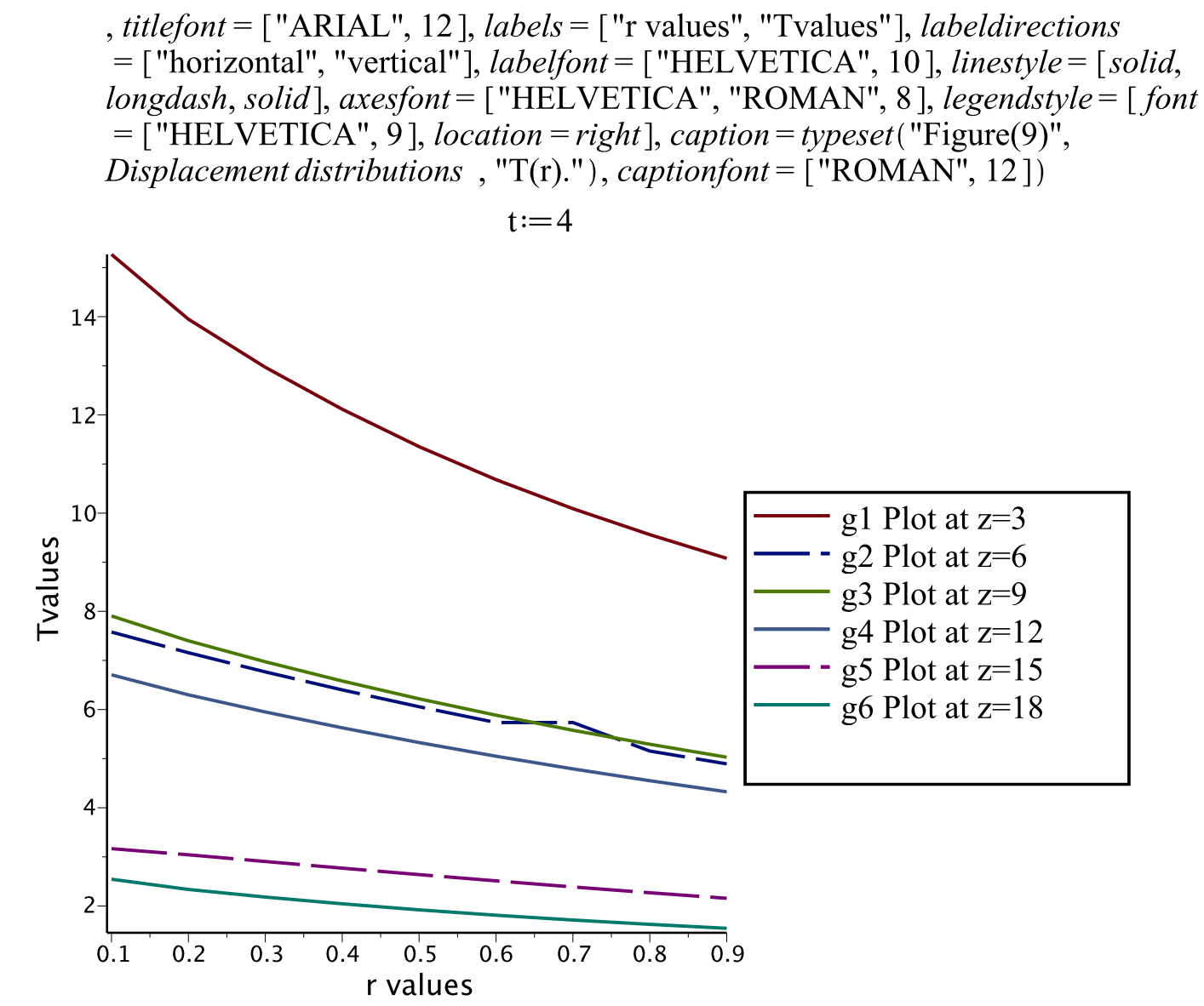

Figure(9)Displacement distributionsT(r).

Using the precedent table we construct curves of spread heat for $\mathrm{U} 1=\mathrm{U} 1(\mathrm{z})$ for circles $(z=3, z=6$, $z=9, z=12, z=15, z=18)$ :

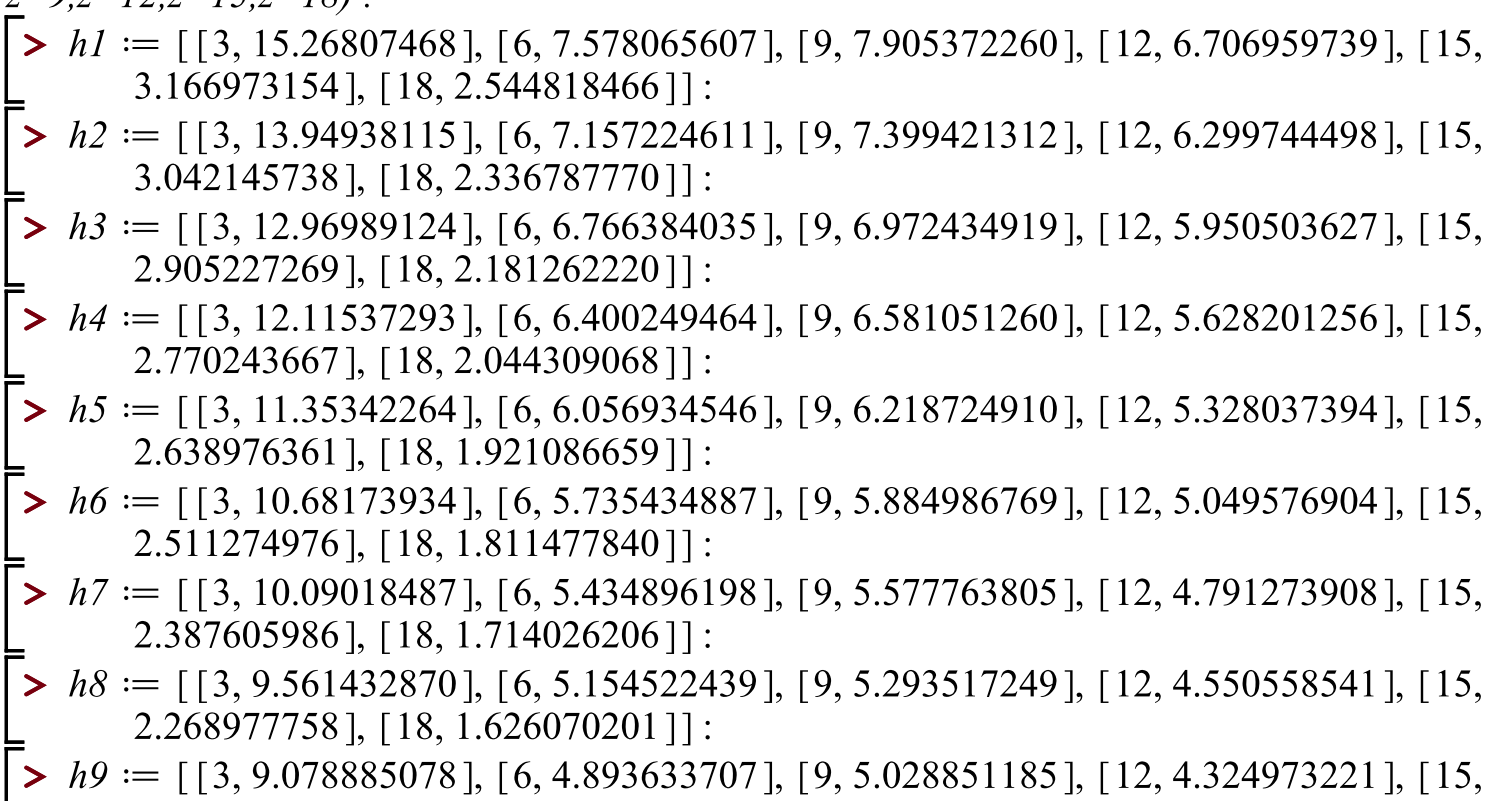




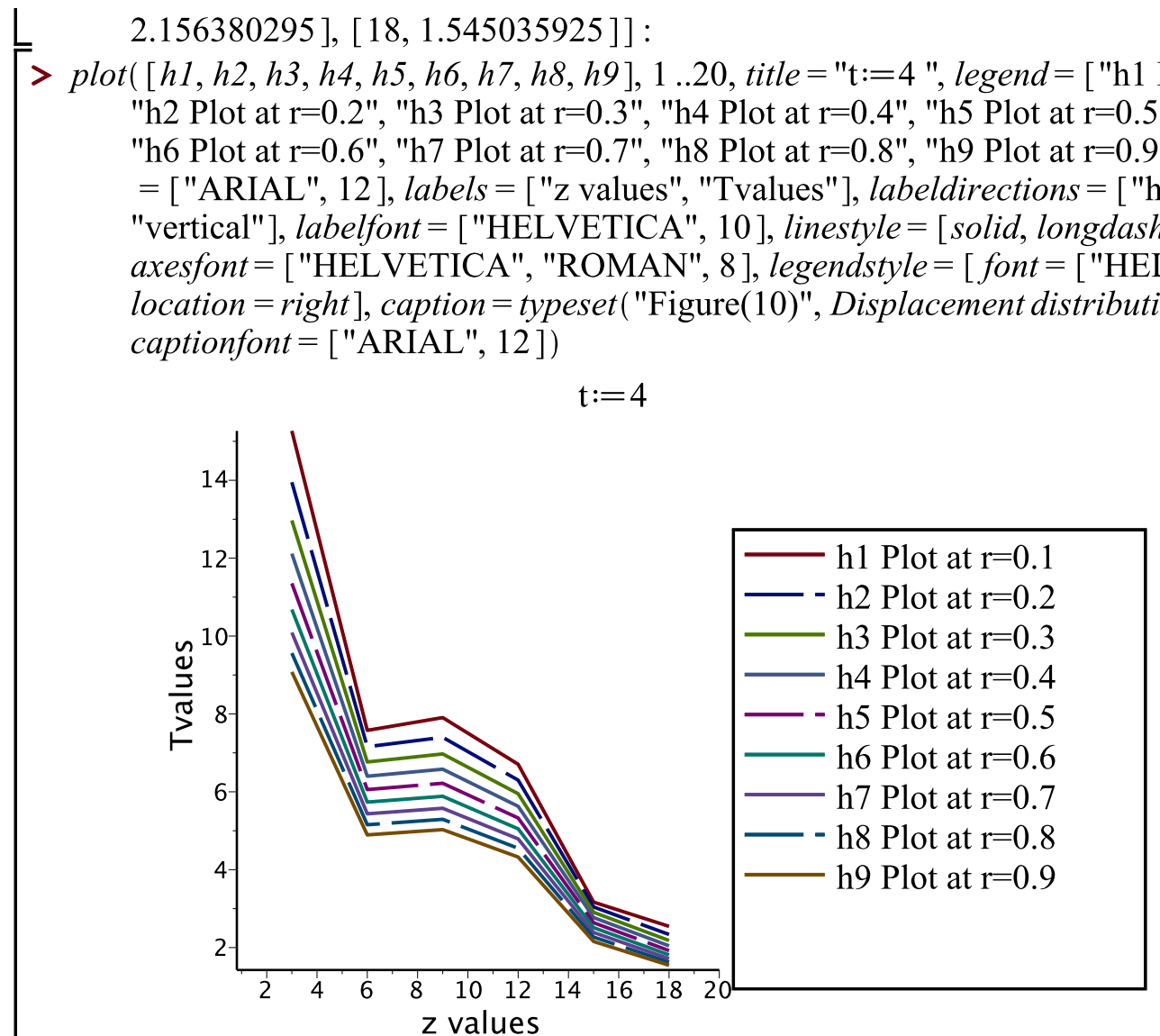

Figure(10)Displacement distributions $\mathrm{T}(\mathrm{z})$.

Comparing figure (9) and figure (10), it can seen easily that the curves are the different trend as the structure is different.From figure(10) it can be noticed that the curves distribution are very close to some. 


\section{Conclusion}

In this paper, the spread heat in nonhomogeneous moving entire cylinder is studied, the solution behavior with discontinuous boundary conditions of the boundary problem is given as an infinite series, using two sequences of integral transformations, Bessel functions theory, we get the solution to the problem of the spread heat in the form of the series, and with writing special programming in Maple program as for a special cases,we constructed to a spread heat as a function of $r$ as a function of $z$.A numerical solutions were obtained and tables as curves defined in $2 \mathrm{D}, 3 \mathrm{D}$. It can be noticed that the inner surface of the cylinder under the effect of two waves be a convex curve and direction from the top to the bottom, as temperatures regularly drop.As for the cylinder which be under the effect one wave, be a convex curve and direction to top. And also through the comparison can be seen that the curves are the different as structure is different.This problem has numerous engineering applications, such as,machining, welding, grinding,are all others practical examples.

\section{Acknowledgements}

The authors are very much grateful to Prof. Ahmed M. K. Tarabia and Dr. R. Tantawy members of mathematics department at Damietta university, Egypt for their valuable comments to bring the paper in the present form.

\section{References}

[1] G. N. Watson, A Treatise on the Theory of Bessel Functions, $2^{\text {nd }}$ edition, Cambridge University Press, Cambridge (1966).

[2] H. S. Carslaw and J. C. Jaeger, Conduction of Heat in Solids, $2^{\text {nd }}$ edition, Oxford University Press, London, England, UK, (1959).

[3] M. N. Özisik, Heat Conduction, $2^{\text {nd }}$ edition, John Wiley and Sons, New York (1993).

[4] G. A. Articolo, Partial Differential Equations and Boundary Value Problems with Maple, $2^{\text {nd }}$ edition, Academic Press, San Diego (2009).

[5] N. S. Koshlyakov,et al, Equation in Partial Mathematical Physics, Visshaya Shkola, Moscow (1970).

[6] G. R. Gasimov, Z. A. Abusutash, Heat Conduction Equation in Physically Inhomogeneous Moving Composite Solids, International Journal of Differential Equations and Applications, AP, LTD, V.14, N.3, (2015),252-270.

[7] A. M. Shahout, G. R. Gasimov and E. A. Rzayev, Solution problem of heat conduction in the circular hollow cylinder using the program Maple11, The Second Conference on Mathematical Sciences, Zarqa Private University, Jordan, (October 22-23,2008), 63-73. 
[8] V. Y. Lotarev, Temperature filed of finite continuous moving cylinder, J. New of Tula State University, V.5, N.2, (1999), 82-86.

[9] S. Y. Kholodovskii, Solving Boundary Value Problems in Cylinders with Multilayer Film inclusion, J, Scientific Notes of Zabaikalskii State University, N. 3, (2012), 145-151. 